\title{
Modeling of transport and technological processes of cultivation and harvesting agricultural crops
}

\author{
Nikolay Maystrenko ${ }^{1}$, Alexandr Levshin ${ }^{1}$, Dmitriy Khort ${ }^{2}$, and Alexey Kutyrev²,* \\ ${ }^{1}$ Russian State Agrarian University - Moscow Timiryazev Agricultural Academy (RSAU - MTAA or \\ RSAU - MAA named after K.A. Timiryazev), Moscow, 127434, Russia. \\ ${ }^{2}$ Federal Scientific Agroengineering Center VIM, 1-st Institutsky proezd, 5, Moscow, 109428, Russia.
}

\begin{abstract}
The productivity of technological processes in agriculture is determined by the set of transport and technological means used. The article describes the models for the unification of shift performance, taking into account a wide range of agricultural cargoes and a large number of works on their movement in the process of cultivation and harvesting of agricultural crops. A step-by-step modeling of scenarios for the use of transport and technological means was carried out on the basis of the optimality criterion and forecasting the period of agricultural work. They confirmed that the objective function is of theoretical and practical interest, as it helps to determine the optimal values of the power of technical means, depending on the combination of production conditions. The numerical value of power obtained by calculation can serve as a guide for manufacturers of agricultural machinery in the development of promising vehicles for a specific consumer, depending on the availability of resources and production and economic conditions.
\end{abstract}

\section{Introduction}

In agricultural production, during cultivation and harvesting, it is customary to distinguish transport and technological processes with appropriate methods for standardizing work. The peculiarity of the processes lies in the sequential execution of cyclic operations that ensure the transportation of technological material to the point of destination - directly or with its preliminary collection (subsequent distribution) on the field [1]. The implementation of technological processes for the cultivation of agricultural crops requires the use of modern, promising technical means of mechanizing individual stages of the production cycle. Today, there is a tendency in the modernization of agricultural machines, as well as the formation of concepts for new types of equipment, and as a result, design with subsequent elements of release into production [2]. Along with this, research is underway in the field of changing energy sources, power plants, for example, a tractor with an electric motor. For the operation of technical means, in the implementation of mechanized work related to the production of crop products that meet the criteria of optimality, mathematical modeling of

* Corresponding author: alexeykutyrev@gmail.com 
production processes is required, taking into account a systematic approach [3,4]. For this purpose, precise numerical values of the operational parameters of transport and transporttechnological units are required, to determine them and conduct an operational and technological assessment, which carries additional time and material costs. In this regard, the purpose of the study is the development, modeling of the operational performance of transport (TS), transport, and technological means (TTS) in the implementation of mechanized work in crop production.

\section{Materials and Methods}

We used a systematic approach to the optimization technique. In accordance with the principle of unification of calculations by excluding conversion factors of different dimensions of the expression for determining the performance of energy resources:

$$
\begin{gathered}
W_{T C}=Q \cdot V_{\Gamma} \cdot \tau / L_{\Gamma} \\
W_{T T C}=Q \cdot V_{\Gamma} \cdot \tau \cdot \mu / L_{\Gamma}
\end{gathered}
$$

where $W_{T C}$ - vehicle capacity, $\mathrm{t} / \mathrm{h}, W_{T T C}$ - productivity of transport and technological means, t / h, $Q$ - cargo capacity, t, $V_{\Gamma}$ - speed of movement on the road with a load, $\mathrm{km} / \mathrm{h}$, $\tau$ - shift working time utilization rate, $\mathrm{L}_{\Gamma}$ - distance of transportation of cargo or process material, km, $\mu-$ a coefficient that allows you to adjust the "clean transport" performance taking into account the additional phase of work - " clean work in the field».

The relationship between the productivity of the transport-technological process and the purely transport process, expressed in terms of the correction factor $\mu$, is as follows:

$$
W_{T T C}=W_{T C} \cdot \mu
$$

In the absence of a field phase in the operation of the transport and technological means, $\mu=1$. Therefore, the technological means performs the functionality of only the vehicle, which provides the basis for determining the unified (for these types of energy means) formula:

$$
W=Q \cdot V_{\Gamma} \cdot \tau \cdot \mu / L_{\Gamma}
$$

In the course of studying the optimization of the performance of mechanized work, a generalized parameter is usually chosen as a criterion - the engine power of an energy vehicle. At the same time, the optimal values of this indicator are determined, which correspond to specific combinations of production and agricultural landscape conditions. However, the transport and transport-technological process is always caused by a combination of the values $\mathrm{Q}$ and $\mathrm{V} \mathrm{G}$, on which the energy characteristic of transport, namely the power of the power plant, depends. A priori, these values affect the stages of vehicle design. At the same time, the combined combination of production and agricultural landscape conditions has a wide range, so there is no optimal TS and TTS in different conditions.

To determine the optimal power and parameters of the mobile power means, it is necessary to determine the nature of the influence of the values $\mathrm{Q}$ and $\mathrm{V}_{\mathrm{r}}$.. Moreover, it is impossible to determine the influence of each of them, since they are interconnected as elements of the power balance. We suggest expressing their product from the power balance, taking into account the movement of a car or tractor on the road in the form: 


$$
Q \cdot V_{\Gamma}=\frac{N \cdot \xi_{N}}{g \cdot f \cdot\left(1+\delta_{e}+\delta_{n}+\delta_{q}\right) /\left(\eta_{M} \cdot \eta_{\sigma}\right)}
$$

where $N$ - engine power of the power vehicle, $\mathrm{kW}, \xi_{N}$ - power utilization factor, $g$ acceleration of free fall, $\mathrm{m}^{2} / \mathrm{s}, f$ - coefficient of resistance to movement (including lifting), $\delta_{e}, \delta_{n}, \delta_{q}$ - the mass ratio, respectively, of the power machine $\mathrm{M}_{\mathrm{e}}$, the trailer $\mathrm{M}_{\Pi}$ and cargo in an additional trailer $\mathrm{Q}_{п}$ to the nominal cargo capacity $\mathrm{Q}$ of the main capacity (body), $\eta_{\mathrm{м} \mathrm{и}}$ $\eta_{\sigma}$ - coefficients that take into account power loss in the transmission and wheel slip. We made changes to the equation. The denominator in the formula characterizes the specific energy consumption per unit of cargo displacement $P_{n}$. For the same type of vehicles of different capacities, we get:

$$
P_{N}=g \cdot f \cdot\left(1+\delta_{e}+\delta_{n}+\delta_{q}\right) / \eta_{M} \cdot \eta_{\sigma} \cdot \xi_{N}, \mathrm{H} / \kappa \Gamma
$$

where $P_{\mathrm{N}}$ - specific energy consumption per unit of cargo movement, $\mathrm{N} / \mathrm{kg}$. With this in mind, the formula is converted to the following form:

$$
W=\frac{N \cdot \mu}{P_{N} \cdot L_{\Gamma}} \cdot \tau
$$

This expression allows you to determine the performance of both transport and process units.

\section{Results and Discussion}

The given mathematical calculations made it possible to determine the operational performance as a function of the characteristics of the external conditions of the process and the power of the technical means. Taking into account the Cob correction factor for local conditions, the expression of the objective function $\mathrm{W}$ is written as the optimization objective function:

$$
W=\frac{N \cdot \mu}{P_{N} \cdot L_{\Gamma}}(h-d N) \cdot K_{O B}
$$

We illustrate the calculated stages of determining the operational performance indicators using the algorithm for the vehicle and TTS in the implementation of mechanized works related to the transportation of agricultural goods, technological material, as well as in the collection and distribution processes (Fig. 1).

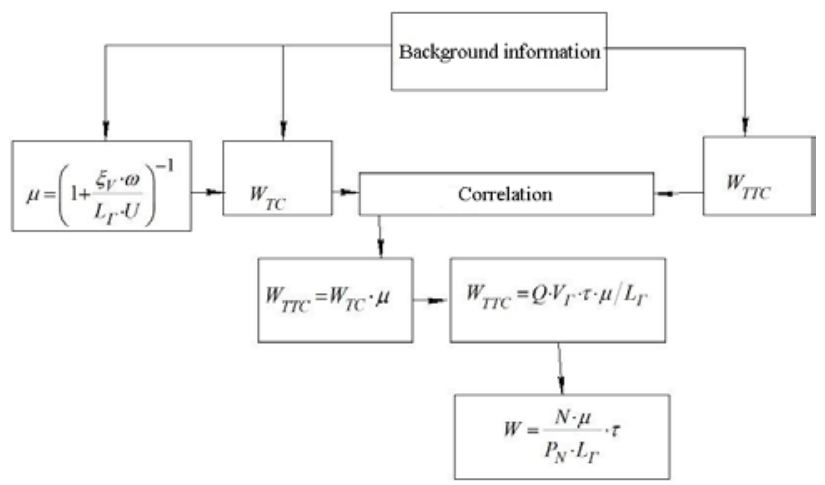

Fig. 1. Unified performance detection algorithm 
These aspects of the mathematical model allow us to determine the formula of operational performance as a function of the characteristics of the external conditions of the process and the power of the technical means. Taking into account the correction factor of the Cob for local conditions, we get:

$$
W=\frac{N \cdot \mu}{P_{N} \cdot L_{\Gamma}}(h-d N) \cdot K_{O B}
$$

where $K_{\text {об }}$ - correction factor, h-coefficient characterizing the loss of shift time, depending on the engine power, $\mathrm{d}$ - coefficient characterizing the loss of shift time, independent of the engine power. Using the performance formula as an objective function (criterion $\mathrm{W} \rightarrow \max$ ), the differentiation method can be used to derive an expression for determining the optimal power $\mathrm{N}_{\mathrm{w}}$. The block diagram of the algorithm for calculating the performance indicators of mobile vehicles and vehicles is shown in Figure 2.



Fig. 2. Block diagram of the algorithm for calculating the performance indicators TS and TTS

The subsequent stage of modeling is characterized by the definition and transformation of the expanded expression of the shift time balance:

$$
T_{C M}=T_{\Pi C}+T_{T O}+T_{3 O}+T_{O C}+T_{X \Gamma}+T_{X P}+T_{H O}
$$

As a result, we obtain an expression of the shift time utilization coefficient $\tau$, which is functionally dependent on the generalized parameter $\mathrm{N}$ in the form of a descending straight line $\tau=h-d N$. The coefficients in the formula are represented by the following algebraic relations between numbers and quantities:

$$
h=1-\frac{t_{C \Pi}+t_{C B}+t_{C J}+t_{T O C}+\left(t_{X \Gamma C}+t_{3 O C}+t_{H O C}\right) \cdot n_{1}}{T_{C M}}
$$




$$
d=\frac{S_{T O} \cdot \theta_{T O}}{T_{C M}}+\left[\begin{array}{c}
\frac{1,25 \cdot K_{Q}}{W_{\Pi}}+0,9 \cdot L_{\Gamma} \cdot K_{V}+K_{X} \cdot \theta_{X} \cdot \beta_{X}+ \\
+\frac{K_{Q} \cdot\left(L_{\Gamma}+0,5 \cdot \omega / U\right)}{q} \cdot\left(\frac{t_{O T}}{l_{O T}}+\frac{t_{H T}}{l_{H T}}\right)
\end{array}\right] \cdot \frac{n_{1}}{T_{C M}}
$$

Each of the coefficients - a parameter of the use of funds, characterizes the relative weighted average loss of shift time.

Based on the obtained data, a program was developed for determining the coefficients of shift time utilization, with the help of which the value of operational productivity is further determined, in accordance with Figure 3.

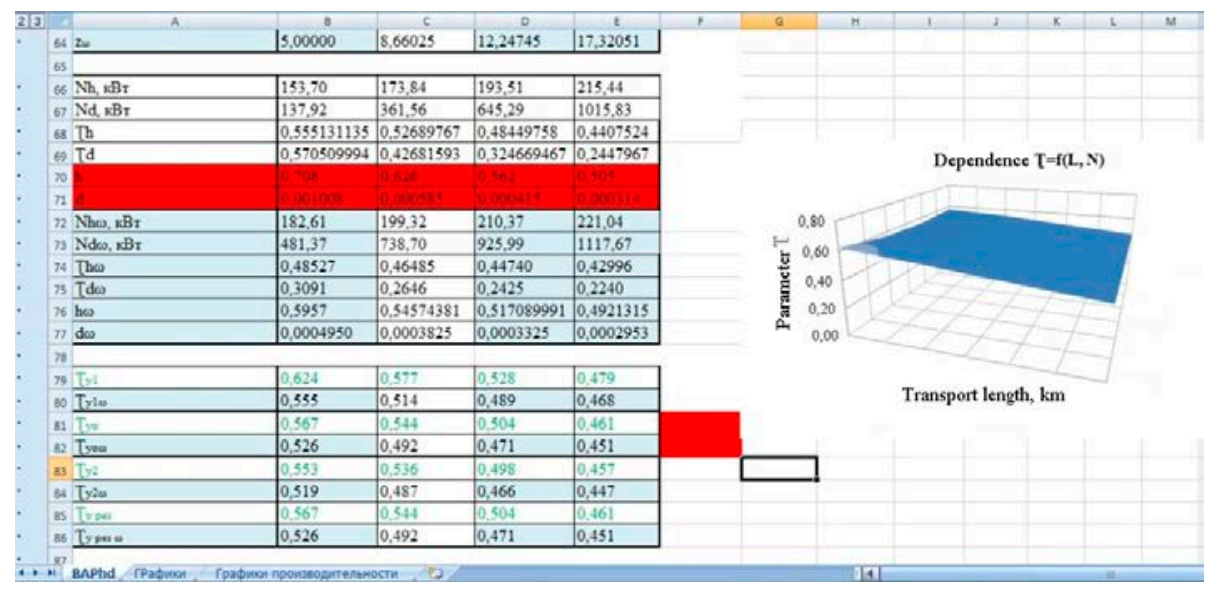

Fig. 3. Values of shift time utilization factors

As a result of the implementation of a more convenient algorithm for calculating and converting the mathematical apparatus, the output values for vehicles during the transportation of mineral fertilizers to the place of their application were obtained.

Car Ural-432065 with a dump truck. The shift time utilization factor was 0,24. Taking into account the value of $\tau$, the operating capacity of 9,1 t/h was calculated. MTZ-82.1 tractor with 2 PTS-6 trailer. The shift time utilization rate was 0,37 . The operating capacity is $6,3 \mathrm{t} / \mathrm{h}$.

We will determine the operational performance for transport and technological means when applying mineral fertilizers using direct-flow technology. Ural-432065 car with Amazone spreader. The time utilization factor is 0.52 . Taking into account the obtained value, the operating capacity of $5.5 \mathrm{t} / \mathrm{h}$ is found. MTZ-82,1 tractor with a RUM-6 spreader. The shift time utilization factor is 0,49 . The operating capacity is $3,9 \mathrm{t} / \mathrm{h}$.

\section{Conclusions}

As a result of the conducted research, the acceptability of the formula describing the linear dependence of the operational performance of technical means on their power is proved. For new or projected vehicles, in the absence of their operational and technological assessment, the production rate can be determined with sufficient confidence by extra - and interpolation or approximation methods based on their calculated performance. Sufficient reliability is confirmed by the correspondence when comparing the obtained calculated values of operational indicators with reference data. As a result of the comparison, it was determined that when performing tractor-transport operations, namely, the delivery of mineral fertilizers (class I cargo) to storage facilities, the operational productivity is $5.84 \mathrm{t}$ / 
$\mathrm{h}$, the obtained theoretical value is $6.3 \mathrm{t} / \mathrm{h}$. In this case, their discrepancy will be $4.2 \%$, which is acceptable for engineering calculations.

\section{References}

1. Khodakarami M., Mitchell K.N., Wang X.B. Modeling Maintenance Project Selection on a Multimodal Transportation Network. Journal of the Transportation Research Record Board, 2409, pp 1-8 (2014).

2. K.T. Malladi, T. Sowlati Optimization of operational level transportation planning in forestry: a review. International Journal of Forest Engineering. 28, pp 198-210, (2017)

3. R.D. MacKinnon, G.M. Barber Optimization Models of Transportation Network Improvement. Progress in Human Geography. 1, pp 387-412, (1977)

4. Ross D. MacKinnon, M. Gerald Barber Optimization Models of Transportation Network Improvement. Progress in Human Geography, 1(3), pp 387-412, (1977) 